\title{
miR-29a in Exosomes from Bone Marrow Mesenchymal Stem Cells Inhibit Fibrosis during Endometrial Repair of Intrauterine Adhesion
}

\author{
Qingqing Tan ${ }^{1}$, Dandan Xia ${ }^{1}$, Xiaoyan Ying ${ }^{2}$ \\ ${ }^{I}$ Department of Gynecological Oncology, The Affiliated Changzhou Maternal and Child Health Care Hospital of Nanjing Medical \\ University, Changzhou, China \\ ${ }^{2}$ Department of Gynecology, The Second Affiliated Hospital of Nanjing Medical University, Nanjing, China
}

Background and Objectives: Bone marrow mesenchymal stem cells (BMSCs) is an ideal source of stem cells in the treatment of intrauterine adhesion. Exosomes are a type of membrane vesicle and the diameter is $30 \sim 100 \mathrm{~nm}$. Exosomes can take their contents into the target cells, releasing and exerting their functions. In this study, we intend to study the role of human BMSC-derived exosomes (BMSC-Exo) in promoting endometrial damage repair in the treatment of IUA.

Methods: We used the magnetic bead affinity method to extract BMSC-Exo and analyzed its biological character. Then we co-cultured the BMSCs-Exo with endometrial cells to detect its effect. We injected BMSCs-Exo into the IUA mouse model. We over-expressed miR-29a in BMSCs-Exo by transient transfection, then used RT-PCR to analyze the expression of the related genes.

Results: BMSCs-Exo expressed exosome-specific proteins CD9, CD63, and CD81. BMSCs-Exo could bring the contents into the target cells. BMSCs-Exo can promote endometrial repair in vitro or in vivo. BMSCs-Exo overexpressing miR-29a can reduce $\alpha$ SMA, Collagen I, SMAD2, and SMAD3.

Conclusions: In this study, we successfully isolated BMSCs-Exo and proved its character and biological activity. BMSCs-Exo can promote cell proliferation and cell migration in vitro and can repair damaged endometrium in the IUA model. The presence of miR-29a in BMSCs-Exo may be an important factor in its resistance to fibrosis during endometrial repair of IUA. This study provides new ideas for the treatment of patients with IUA and has important clinical research significance.

Keywords: Bone marrow mesenchymal stem cells, Exosomes, miR-29 $\alpha$, Anti-fibrosis

Received: March 26, 2020, Revised: July 25, 2020,

Accepted: August 23, 2020, Published online: November 30, 2020

Correspondence to Xiaoyan Ying

Department of Gynecology, The Second Affiliated Hospital of Nanjing Medical University, 121 Jiangiiayuan, Nanjing 210011, China Tel: +86-02583348750, Fax: +86-02558509994

E-mail: xiaoyanying1516@hotmail.com

(c) This is an open-access article distributed under the terms of the Creative Commons Attribution Non-Commercial License (http://creativecommons.org/ licenses/by-nc/4.0/), which permits unrestricted non-commercial use, distribution, and reproduction in any medium, provided the original work is properly cited.

Copyright (c) 2020 by the Korean Society for Stem Cell Research

\section{Introduction}

Intrauterine adhesion (IUA) also known as Asherman syndrome, is caused by fibrosis of the endometrium. The uterine cavity of the patient is partially or completely occluded, which subsequently causes oligomenorrhea, amenorrhea, and insufficiency pregnancy or recurrent miscarriage $(1,2)$. With the increase of uterine invasive procedures such as abortion and various reproductive tract infections, especially the induced abortion has been increasing, the incidence of IUA has also gradually increased (3). This severely affects women's reproductive function. The 
researchers have done a lot of studies on the pathogenesis of IUA and believe that endometrial repair disorders may be the main mechanism of IUA formation (4-6). There are some problems in the treatment of IUA, especially in patients with a severe or extensive endometrial injury. The treatment is still difficult to solve the problem of endometrial regeneration (7). Therefore, it is of great significance to explore new and effective treatments, restore the normal tissue structure and function of the endometrium, and improve the uterine fertility function of patients with IUA.

In recent years, with the deepening of the understanding of stem cells, the use of hysteroscopic surgery combined with stem cells to treat IUA has become a new therapeutic way. Stem cells are a class of undifferentiated cells with self-replication ability and multi-directional differentiation potential. This characteristic makes them embody important values in experimental research and clinical treatment applications (8-10). Bone marrow mesenchymal stem cells (BMSCs) are a type of adult stem cells with multi-directional differentiation potential. It is an ideal source of stem cells in tissue engineering (11). BMSCs are also currently used in the treatment of IUA $(12,13)$. Although BMSCs have some effect on the treatment of IUA, there are some problems such as immune rejection, cell stability, and ethical issues. Also, the mechanism by which transplanted BMSCs promote endometrial regeneration remains unclear. Therefore, it is necessary to find a new way to solve the problems in the clinical treatment of stem cells.

Recently, more and more researchers have discovered that intercellular communication is a way of transmitting information between cells $(14,15)$. Exosomes are a type of membrane vesicle and the diameter is $30 \sim 100 \mathrm{~nm}$. When exosomes are transported to the target cell, they fuse with the target cell's plasma membrane or are endocytosed into the cell, releasing their contents and exerting their functions (16). Exosomes are mainly composed of proteins, lipids, mRNA, and miRNA. Exosomes derived from different types of cells have different proteins, including common proteins and cell-specific proteins (17-19). Among the biomolecules carried by exosomes, micro non-coding RNAs (miRNAs) have received widespread attention for their important role in regulating gene expression. Therefore, we infer that BMSCs-derived exosomes contain BMSCs-specific proteins, mRNAs, and miRNAs. This enables exosomes to perform functions similar to BMSCs.

In view of this, we intend to study the role of human bone marrow mesenchymal stem cell-derived exosomes in promoting endometrial damage repair in the treatment of IUA. This may provide new methods for endometrial repair in patients with intrauterine adhesion.

\section{Materials and Methods}

\section{Ethics statement}

This study was approved by the Affiliated Changzhou Maternal and Child Health Care Hospital of Nanjing Medical University research ethics committee (No. 2018023). The protocols, including all relevant details, were carried out in compliance with the institutional and national guidelines.

\section{Isolation, culture, and characterization of BMSCs}

Human bone marrow was collected from 3 donors by puncturing. Then diluted the bone marrow with equivalent saline. The diluted bone marrow was added gently on the top of equivalent Ficoll (Sigma-Aldrich, USA), centrifuged the mixture at 2,000 rpm for 30 mins, then extracted the interface layer and washed by saline for 3 times. Cells were inoculated in a dish at a density of $1 \times 10^{6}$. Human BMSCs were cultured by adherent culture. The medium was BMSCs culture medium: containing $10 \%$ fetal bovine serum (Gibco, USA), $2 \mathrm{mmol} / \mathrm{L} \mathrm{L-gluta-}$ mine (Gibco, USA), $0.1 \mathrm{U} / \mathrm{L}$ penicillin (Gibco, USA), and $0.1 \mu \mathrm{g} / \mathrm{L}$ Streptomyces (Gibco, USA) in the low-glucose DMED medium (Gibco, USA). BMSCs were cultured at $37^{\circ} \mathrm{C}$ and $5 \% \mathrm{CO}_{2}$. After the primary BMSCs are adhered to for 3 days, remove the non-adherent cells by rinse the culture flask with PBS, then add the complete BMSCs culture medium, and change the medium every $1 \sim 2$ days. The morphological characteristics of BMSCs were observed under the microscope.

\section{Flow cytometry analysis}

To characterize the BMSCs, 3rd passage cells were trypsinized and re-suspended in phosphate-buffered saline (PBS), with $1 \%$ bovine serum albumin (BSA). The cells were incubated at $4^{\circ} \mathrm{C}$ for $1 \mathrm{~h}$ with primary antibodies against CD29, CD44, CD73, HLA-DR (eBioscience, San Diego, CA); CD90, CD105, and CD45 (Biolegend, San Diego, CA); CD34 (BD Pharmingen, San Diego, CA). Proper isotype antibodies were used as control. Cells were then washed twice with PBS containing 1\% FBS and resuspended in $0.5 \mathrm{ml}$ PBS. Quantitative flow cytometry analysis was performed using a FACScan flow cytometer (Beckman Coulter, CA). 


\section{Exosome derived from BMSCs}

We used a magnetic bead affinity method to extract exosomes. After removing the medium, the BMSCs cells were rinsed 3 times with PBS to completely remove the serum components from the medium. It was then cultured overnight in low-glucose DMEM medium without serum, and the supernatant was collected. The obtained supernatant was centrifuged at $2,000 \mathrm{~g}$ for $20 \mathrm{~min}$ to removed cell debris. Subsequently, the obtained cell-free supernatant was concentrated using a Millipore ultrafiltration tube $100 \mathrm{~K}$, and the supernatant was concentrated to less than $1 \mathrm{ml}$ to obtain exosomes more efficiently. Then we used MagCapture ${ }^{\mathrm{TM}}$ Exosome Isolation Kit PS (FUJIFIL $M$ Wako, Japan) to isolate exosomes according to the manufacturer's instructions. We stored the exosome at $-80^{\circ} \mathrm{C}$ for further use.

\section{Definition of exosome concentration in this study}

In order to use the exosomes more conveniently, we designed exosomes from cells in a $10 \mathrm{~cm}$ diameter dish as one unit. According to the detection in Fig. 2B, we have known the concentration of one-unit exosomes was $4.1 \mathrm{E}+$ 9 Particles/ml, then we can figure out the concentration of exosomes used in other tests. If there are no special instructions, the concentration of exosomes used in the test was one-unit in this study.

\section{Western blot characterization of exosome}

We added $5 \times$ loading buffer (Thermo Scientific, UK) into the samples, and cooked at $95^{\circ} \mathrm{C}$ for $5 \mathrm{~min}$. Then we added the samples into the SDS-PAGE gel (Bio-Rad, USA), performed $1.5 \mathrm{~h}$ electrophoresis at $150 \mathrm{~V}$ constant pressure. After the electrophoresis, we transferred the gel to the nitrocellulose membrane (Bio-Rad, USA). We incubated the membrane with CD9 (Abcam, USA), CD63 (Abcam, USA) and CD81 (Abcam, USA) antibodies at $4^{\circ} \mathrm{C}$ overnight. Then incubate the membrane with horseradish peroxidase-labeled goat anti-rabbit secondary antibodies (Abcam, USA) for $30 \mathrm{~min}$ at room temperature. We used the chemiluminescence reagent as a substrate and imaging.

\section{Exosome activity test}

We used PKH26 dye (Sigma-Aldrich, USA) to interact with exosomes. According to the kit instructions, we took $100 \mu 1$ of BMSCs-Exo suspension and resuspend in Diluent $\mathrm{C}$ solution. We added another $1 \mu 1$ of PKH26 dye to $250 \mu 1$ Diluent C solution, and quickly mix. After 5 minutes of incubation at room temperature, an equal volume of fetal calf serum was added to stop the reaction.
We rinsed twice with low glucose DMEM medium $(100,000 \mathrm{~g}, 2 \mathrm{~h})$, and then added cell basal medium to resuspend. We took fibroblasts with good growth status, added PKH26-labeled exosomes during the culture process, and observed the effects at different time points under a microscope.

\section{Exosome co-culture with endometrial epithelial cells}

We isolated endometrial epithelial cells from 8 weeks old estrous female ICR mouse. Endometrial epithelial cells in good growth conditions were used to co-culture with exosomes. We digested the cells with $0.25 \%$ trypsin and added a complete culture solution to make a cell suspension. Then we adjusted the cell density after counting, inoculated $1 \times 10^{4} / \mathrm{ml}$ into 96-well plates, $100 \mu \mathrm{l} /$ wells. BMSCs-derived exosomes were added into the cells. After the exosomes and cells had fully functioned, the cell growth curve was drawn using the CCK-8 method with time as the abscissa and the absorbance values (OD values) at different time points as the ordinate.

\section{Establishment of a mouse model of intrauterine adhesion}

We used 8 weeks old estrous female ICR mouse to do the surgery. We anesthetized mouse with an intraperitoneal injection of $5 \%$ chloral hydrate. The dosage of chloral hydrate was $0.1 \mathrm{ml} / 10 \mathrm{~g}$. After anesthesia, the mouse's abdominal cavity was opened through a median incision in the lower abdomen, and the "Y-shaped" mouse uterus was found behind the bladder. The right uterus was selected as the treatment side and the left uterus was used as the self-control. We used two vascular clamps respectively clamp the upper right uterine horn and lower Y-shaped uterine bifurcation. Then we used a $1 \mathrm{ml}$ syringe to insert the uterine cavity and injected $0.1 \mathrm{ml}$ of ethanol. According to the grouping requirements, they will stay in the uterine cavity for $10 \mathrm{~s}$. When the time was up, the ethanol was drawn out. Finally, we loosened the vascular clip, flushed the abdominal cavity with saline, and closed the abdomen layer by layer.

For exosomes treatment, the surgery was done as described above. One-unit exosomes were injected into the uterine cavity for each mouse.

\section{Histological analysis}

The damaged uterus were collected and fixed with $4 \%$ concentration of paraformaldehyde overnight. The tissues were dehydrated in stratified alcohols and then embedded in paraffin. The samples were sliced consecutively into sections with 5 um thickness and normally stained with 
hematoxylin and eosin (HE). The sections were observed under a light microscope.

\section{RNA isolation and RT-PCR}

Total RNA was isolated using the RNeasy Mini Kit (Qiagen, UK) following the manufacturer's protocol. The quality and quantity of RNA were checked on a NanoDrop spectrophotometer (Thermo Scientific, UK). A total of 1 $\mu \mathrm{g}$ RNA was reverse transcribed into cDNA. RT-PCR was performed by using SYBR Green PCR reagent (Thermo Scientific, UK). GAPDH was used as an internal reference. Normalization and fold changes were calculated using the comparative CT $(2-\Delta \Delta \mathrm{Ct})$ method. The average values were obtained from three individual experiments. The primer sequences are shown in Table 1.

\section{Overexpression of $\mathrm{miR}-29 \mathrm{a}$ on BMSCs}

In this study, we used the transfection reagent Fugene HD (Promega, USA) to transiently transfect miR-29a on BMSCs. The miR-29a was designed and composited by the company. We made the Fugene HD-miR-29a complex according to the manufacturer's instruction. The complex was added to the BMSCs. We collected the supernatant after culturing $48 \mathrm{~h}$ and then extracted the exosomes.

\section{Exosomal miRNA extraction}

The exosome miRNA was isolated via the ultracentrifugation method as reported in the previous study (20). We collected the BMSCs-Exo from the conditioned medium according to the procedure mentioned above. Thereafter, RT-PCR was performed as described above for the miR-29a level.

\section{Statistical analysis}

Statistical analysis was performed with GraphPad Software (San Diego, CA). Analysis of variance followed by Student's t-test was applied to determine the significant differences between groups. $\mathrm{p}<0.05$ were considered as statistically significant. All data were presented as the mean \pm SD.

\section{Results}

\section{Identification of BMSCs}

BMSCs were able to grow adhering to the wall, and cells are fusiform or irregularly distributed. After 3 days of cultured, the number of cells gradually increased and arranged closely. After 6 days of cultured, the growth of the cells can cover $80 \%$ of the entire culture flask (Fig. 1A).

To detect the proliferation capacity of BMSCs after cultured in vitro, we took the third-generation and fifth-generation cells in a good growth state, digest with $0.25 \%$ trypsin and add complete culture solution to make a cell suspension, adjust the cell density after counting, and inoculate $1 \times 10^{4} / \mathrm{ml}$ in 96 -well plates. The cell growth curve was drawn using the CCK- 8 method. It can be seen from the growth curve that the cells began to enter the logarithmic growth phase on the second day after inoculation, and reached the plateau phase on the sixth day, the cells appeared growth inhibition after the eighth day. The proliferative capacity of the 5th passage cells is significantly lower than that of the 3rd passage cells (Fig. 1B). So, we used the 3rd generation cells for subsequent experiments.

Next, we examined the expression of molecules on the surface of BMSCs by flow cytometry. The results showed that BMSCs expressed mesenchymal cell surface markers CD29, CD44, CD73, CD90, and CD105, did not express MHC-II molecules HLA-DR, and did not express hematopoietic stem cell markers CD34, CD45 (Fig. 1C). This is consistent with the previous report (9).

\section{Isolation and identification of BMSCs derived exosomes}

We used magnetic beads and phosphatidylserine (PS) binding protein (PS affinity method) to extract exosomes derived from BMSCs (BMSCs-Exo). The BMSCs-Exo were collected and observed by transmission electron microscopy. As shown in the Fig. 2A, BMSCs-Exo showed a clear saucer-like shape under the observation of the transmission electron microscope. Next, the obtained exosomes were tested for particle size. From the Fig. 2B, we can see that the particle size of the obtained exosomes is about

Table 1. Primer sequence in this study

\begin{tabular}{lll}
\hline \multicolumn{1}{c}{ Genes } & \multicolumn{1}{c}{ Forward primer sequence } & \multicolumn{1}{c}{ Reverse primer sequence } \\
\hline$\alpha$ SMA & ACTGAGCGTGGCTATTCCTCCGTT & GCAGTGGCCATCTCATTTTCA \\
Collagen I & GAGCGGTAACAAGGGTGAGC & CTTCCCCATTAGGGCCTCTC \\
SMAD2 & CCGACACACCGAGATCCTAAC & GAGGTGGCGTTCTGGAATATAA \\
SMAD3 & CCATCTCCTACTACGAGCTGAA & CACTGCTGCATTCCTGTTGAC \\
GAPDH & TGTGGGCATCAATGGATTGG & ACACCATGTATTCCGGGTCAAT \\
\hline
\end{tabular}


A

$3 \mathrm{~d}$

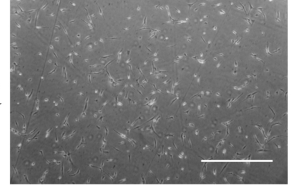

$6 \mathrm{~d}$

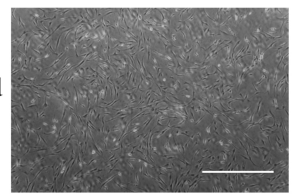

C

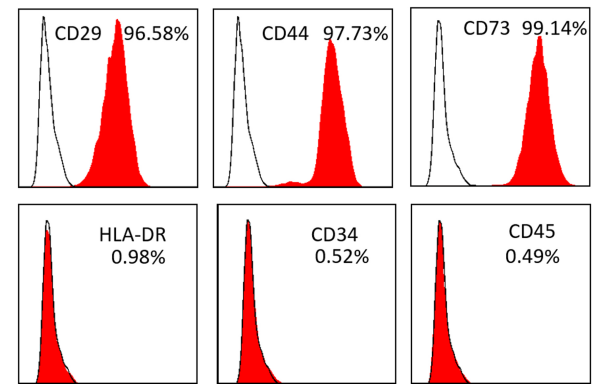

A

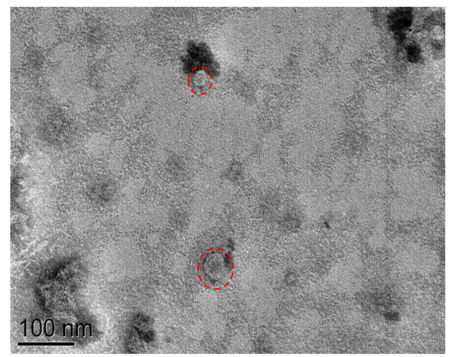

C

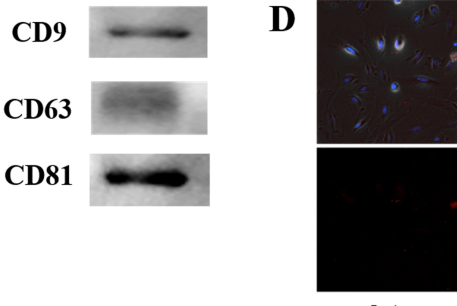

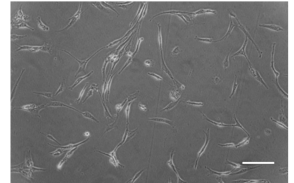
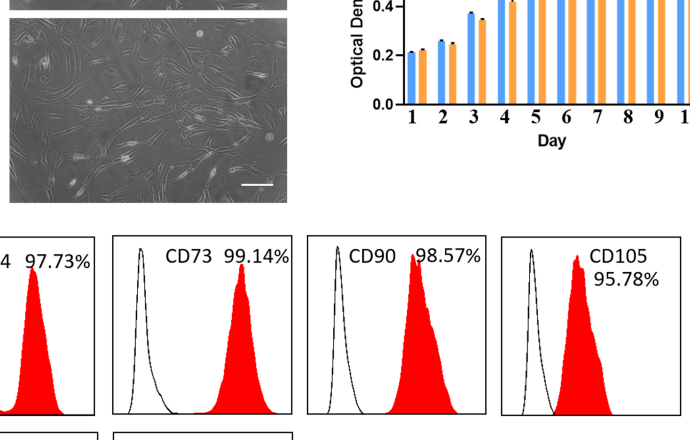

\section{CD45} (1)

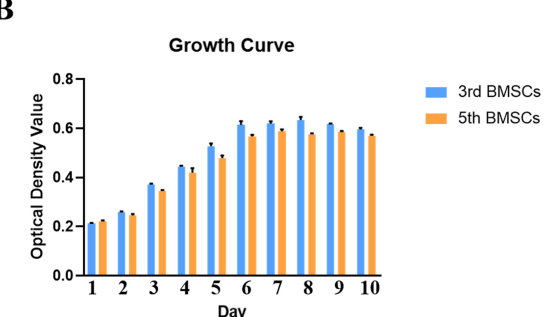

Fig. 1. Culture and identification of BMSCs. (A) Cellular morphology of BMSCs, Scale bar: $40 \mu \mathrm{m}$. (B) Growth curve of 3rd BMSCs and 5th BMSCs. (C) Expression of surface antigens was carried out by flow cytometry using CD29, CD44, CD73, CD90, CD105, HLA-DR, CD34, and CD45.
B
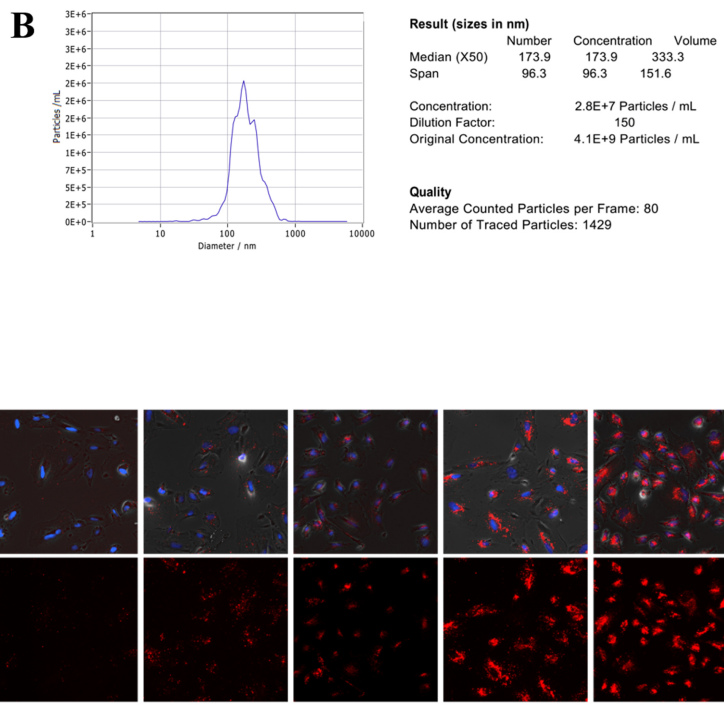

$30 \mathrm{~min}$

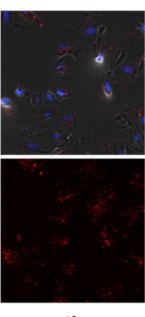

1h

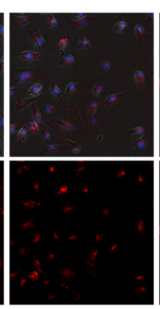

$2 h$

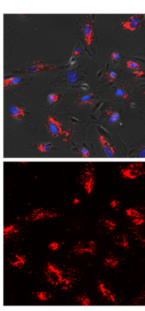

5 h

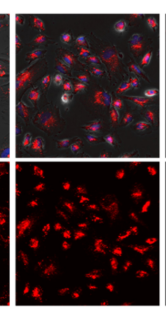

$10 \mathrm{~h}$

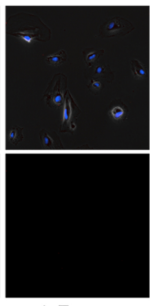

w/o Exosome

Fig. 2. Identification of BMSCs derived exosomes. (A) Transmission electron microscopy analysis of BMSCs-Exo, Scale bar: $100 \mu \mathrm{m}$. (B) Particle size analysis of BMSCs-Exo. (C) Western blot analysis of exosome-specific proteins CD9, CD63, and CD81 in BMSCs-Exo. (D) Immunofluorescent staining analysis to detect labeled exosomes biological activity.

$170 \mathrm{~nm}$ and the concentration is $4.1 \mathrm{E}+9$ Particles $/ \mathrm{ml}$. Subsequently, we used western blot to detect the expression of exosome-specific proteins CD9, CD63, and CD81 (Fig. 2C).

To test whether the obtained exosomes have biological activity, we labeled the exosomes with PKH26 dye, added the labeled exosomes to fibroblasts, and observed the situation of exosomes entering cells at different times. The results showed that over time, the labeled exosomes gradu- ally entered the cells (Fig. 2D). This indicated that the obtained exosomes had biological activity and could bring the contents into the target cells to play a role.

\section{BMSCs-Exo can promote endometrial repair in vitro and in vivo}

To prove the functionality of BMSCs-Exo, we co-cultured the one-unit BMSCs-Exo with endometrial cells for 5 days, then used CCK8 to measure the OD value of the 
cells that acted at different time points. From Fig. 3A, we can see that with the increase of culture time, cells proliferation gradually slows down and enters the plateau phase. The experimental group added BMSCs-Exo had a faster proliferation rate than the control group. These results suggest that BMSCs-Exo can promote the proliferation of endometrial cells.

To test whether the obtained BMSCs-Exo could promote migration, we used a scratch test to verify. When mouse endometrial epithelial cells were overgrown in the 6 well plate, we used the pipette to make a straight scratch on the plate. Then we co-cultured cells with BMSCs-Exo and observed the cell growth of the scratch for 2 days and 5 days. From Fig. 3B, we can see that the experimental group added with BMSCs-Exo was significantly smaller in the width of the scratch than the control group. The scratches had healed in the BMSCs-Exo group by 5th day. These results suggest that BMSCs-Exo can promote cell migration.

We first established a mouse model of intrauterine adhesions and then injected BMSCs-Exo into the mouse model of intrauterine adhesions. After 2 weeks of repair, samples were collected for $\mathrm{HE}$ staining analysis. From Fig. 3C, we can see that the effect of tissue restoration with BMSCs-Exo is better than the control group, which is mainly manifested in the increase in the number of glands, the increase in intimal thickness, and the decrease in fibrosis. We observed the tissue sections under a microscope with high power. Each section follows the order from left to right and top to bottom, observed and counted the endometrial glands, repeated the count 3 times for each section. The number of endometrial glands was shown in Fig. 3D. The above results suggest that BMSCs-Exo can repair damaged endometrium in mice.

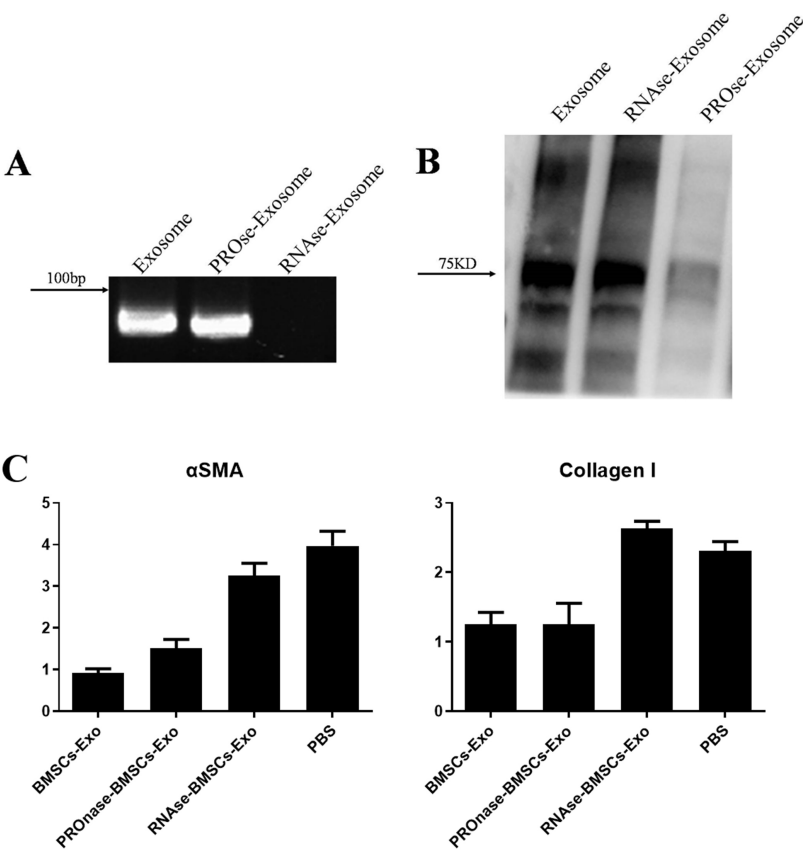

Fig. 4. The nucleic acid component present in BMSCs-Exo can play a role in inhibiting the formation of fibroblasts. (A) Gel electrophoresis of BMSCs-Exo after lysed by proteinase $\mathrm{K}$ or RNase A. (B) Silver nitrate staining of BMSCs-Exo after lysed by proteinase $K$ or RNase A. (C) The expression of fibroblast formation related gene ( $\alpha$-SMA, Collagen I) in TGF $\beta$-induced fibroblasts models, which treated by BMSCs-Exo, RNAse-BMSCs-Exo, PROnase-BMSCs-Exo, and PBS.
A
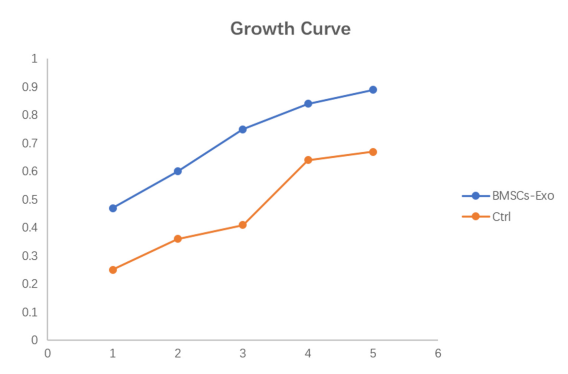

B

PBS

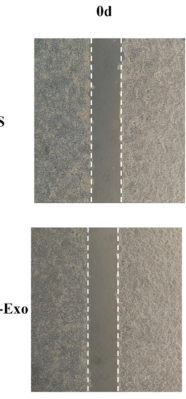

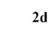
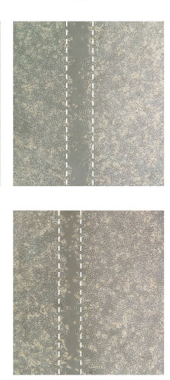

$5 d$

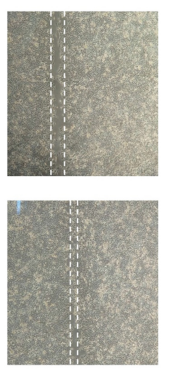

C

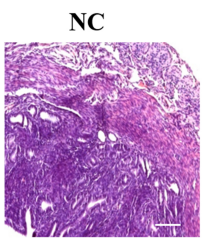

PBS

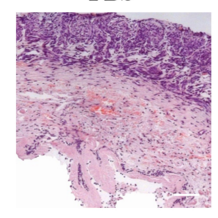

BMSCs-Exo

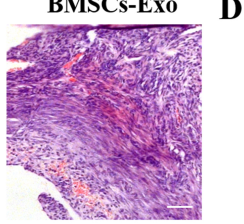

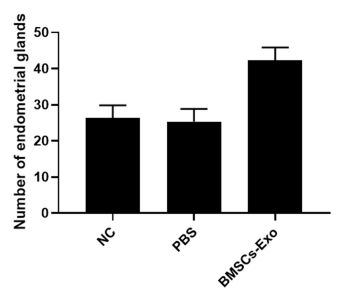

Fig. 3. BMSCs-Exo promotes endometrial repair in vitro or in vivo. (A) Growth curve of endometrial cells co-cultured with BMSCs-Exo. (B) BMSCs-Exo promoted cell migration by scratch test. (C) HE staining analysis to detect the ability of BMSCsExo to repair endometrial damage in vivo, Scale bar: $100 \mu \mathrm{m}$. (D) The number of endometrial glands. 
A

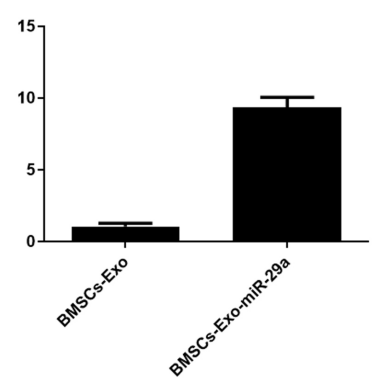

B
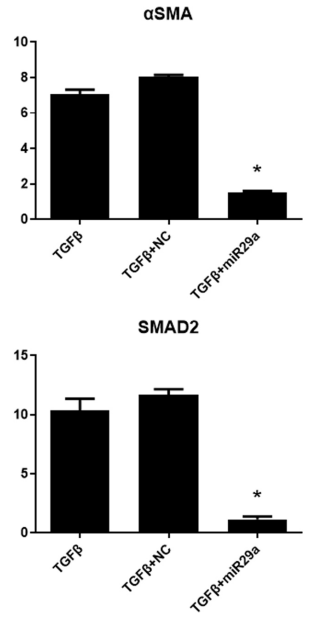

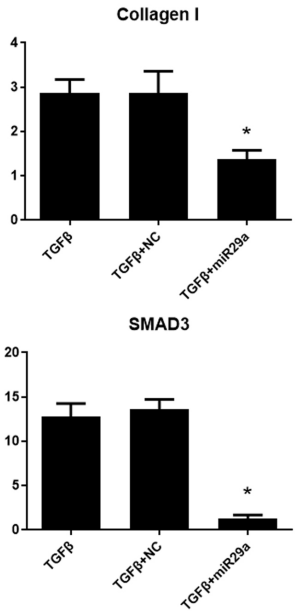

Fig. 5. miR-29a-3p in BMSCs-Exo has an anti-fibrotic role during the repair process. (A) miR-29a overexpressed in BMSCs-Exo used transient transfection. (B) RT-PCR analysis of the expression of fibroblast formation related gene, NC: fibroblastExo, miR29a: BMSCs-Exo overexpressing miR-29a, $* p<0.05$.

\section{miR-29a in BMSCs-Exo has an anti-fibrotic role during the repair process}

Based on the current research, the pathogenesis of IUA has not been indicated, but most experts believe that IUA is a fibrotic lesion when repaired after endometrium damaged (21-23). We used fibroblasts isolated from uterine of 8 weeks old estrous female ICR mouse to detect the effect of BMSCs-Exo. The fibroblasts were plated in a 6-well plate. When the cells grew to $70 \%$ of the whole well, 10 $\mathrm{ng} / \mathrm{ml} \mathrm{TGF} \beta$ cytokines were added to induce the fibroblasts. The induced cells were used in subsequent experiments.

To study molecules in BMSCs-Exo that may influence fibroblast formation, we first treated one-unit BMSC-Exo with $100 \mathrm{ul}$ proteinase $\mathrm{K}$ (PROnase) or $10 \mathrm{mg} / \mathrm{ml}$ RNase A for $2 \mathrm{~h}$. We used agarose gel electrophoresis and silver nitrate staining to detect whether the protein or nucleic acid components in BMSCs-Exo were completely lysed. The results of gel electrophoresis revealed that (Fig. 4A), the nucleic acid components carried by BMSCs-Exo and PROnase-BMSCs-Exo were mainly small fragments of RNA ( $<100 \mathrm{bp}$ ). The proteins in RNAse-BMSCs-Exo and untreated BMSCs-Exo were concentrated at about $75 \mathrm{KD}$, and the expression of exosomes treated with protease decreased significantly (Fig. 4B).

We added BMSCs-Exo, RNAse-BMSCs-Exo, PROnaseBMSCs-Exo, and PBS to TGF $\beta$-induced fibroblasts models, and examined their effects on fibroblast formation. We used RT-PCR to analyze the expression of a related gene. The results showed that the expression of fibroblast-specific markers $\alpha$ SMA and Collagen I in the Exo group treated with RNase was significantly higher than the other two experimental groups and closer to the untreated group. The results indicated that after RNase treatment, BMSC-Exo lost some of its components and thus lost the effect of inhibiting fibroblast production (Fig. 4C). The above results suggested that the nucleic acid component present in BMSCs-Exo can play a role in inhibiting the formation of fibroblasts, but the protein component does not have this function.

Then we used high-throughput sequencing analysis to detect all miRNAs in BMSCs-Exo and sorted them according to their content. Current research indicates that the target genes of miR-29a have the most effect on cell fibrosis-related proteins (24-26). In our study, the content of miR-29a is also among the top, so we chose miR-29a as the target for research. We used transient transfection to make miR-29a overexpressed in BMSCs-Exo and subsequently detected the miR-29a by RT-PCR analysis. It was found that the over-expressed BMSCs-Exo miR-29a content was significantly increased (Fig. 5A), indicating that our over-expressed BMSCs-Exo can be used for the next experiment. Then we isolated and extracted BMSCsExo with high expression of miR-29a, added it to the cell model, and used RT-PCR to analyze the expression of the related genes. As shown in the Fig. 5B, BMSCs-Exo overexpressing miR-29a can reduce $\alpha \mathrm{SMA}$, Collagen I, SMAD2, and SMAD3. The above results suggested that miR-29a in BMSCs-Exo may be an important factor for its anti-fibrosis during endometrial repair of intrauterine adhesions.

\section{Discussion}

Based on the current research, the pathogenesis of IUA has not been specified, but most experts believe that the main pathological changes in the pathogenesis of IUA are as follows (21-23). After the endometrium is damaged, 
there is a local infection or ischemic response in the endometrium at the damaged location, which will promote cells to secrete many factors, and these factors interact and affect the process of repairing the endometrium. The proliferation and migration of fibroblasts maintain the synthesis and degradation of ECM, thereby achieving the role of repair. However, if some cytokines are abnormally expressed during the repair process, the normal regulatory effect will be out of balance, leading to the accumulation of ECM and inhibiting endometrial repair. The new connective tissue will replace the endometrial tissue, causing endometrial fibrosis. Eventually, lead to the occurrence of IUA. IUA is a fibrotic lesion caused by abnormalities when repaired after the endometrium is damaged. So we choose fibroblasts to detect the effect of BMSCs-Exo in vitro.

More and more studies have shown that exosomes secreted by mesenchymal stem cells play a certain role in the treatment of diseases (27-31). The paracrine effect of mesenchymal stem cells has been proven to be one of how it works, and exosomes derived from mesenchymal stem cells may be important substances in this role. Mesenchymal stem cell-derived exosomes have played a good role in a variety of animal disease models. These studies suggest that exosomal treatment can become a new type of clinical treatment, thereby avoiding the problems caused by stem cell therapy. So we chose BMSCs-Exo to study its role in the repair of endometrial damage.

Exosomes are mainly composed of proteins, lipids, mRNA, and miRNA. Exosomes derived from different types of cells have different proteins, including common proteins and cell-specific proteins (17). Exosomes derived from different tissues may have the memory of the tissue, not only with tissue-specific protein molecules, but also with functional molecules $(18,19)$. Therefore, we speculate that BMSCs-Exo contains BMSCs-specific proteins, mRNAs, ncRNAs, miRNAs, and other substances that can perform functions like BMSCs. BMSCs-Exo plays a role as a complex, delivered its contents into target cells to have an effect. It is the focus of our research whether BMSCs-Exo can regulate the target cells through its content and have the ability to resist fibrosis during the process.

Transforming growth factor-beta (TGF $\beta$ ) has attracted more and more researchers' attention due to its important role in embryonic development. Current research confirms that TGF $\beta$ plays an important role in fibrous scar formation. The high expression of TGF $\beta$ at the damaged site may be the main reason for the formation of fibrous scars. Therefore, TGF $\beta$ is currently recognized as the strongest fibrogenic cytokine and can stimulate the formation of extracellular matrix (ECM) (32). TGF $\beta$ combined with its receptor, then transmits signals in the extracellular environment into the cell through the SMAD pathway (33). Previous studies have shown that SMAD2 and SMAD3 are highly expressed during scar hyperplasia and have high levels of phosphorylation. They cannot regulate the formation of fibroblasts and the extracellular matrix Collagen I, which leads to excessive proliferation. Interfering with the expression of SMAD2 and SMAD3 can reduce the formation of fibroblasts and thus reduce scarring (34). Therefore, inhibition of the TGF $\beta$ /SMAD pathway may be a method for treating scar hyperplasia.

Recently, researchers have found that microRNA-29 (referred to as miR-29) is closely related to fibrotic diseases and has gradually become a new focus of research. The study also found that the decline in miR-29 expression was proportional to the degree of fibrosis (35). Previous studies have found that the expression of miR-29 is related to the expression of TGF $\beta, \operatorname{TGF} \beta$ can inhibit the expression of miR-29, and conversely, overexpression of miR-29 can reduce the expression of TGF $\beta$, thereby inhibiting the activity of Smad3, reaching Purpose of antifibrosis (36). These studies show that miR-29 could resist fibrosis, which provides new ideas for the treatment of fibrotic diseases. The currently known miR-29 family includes miR-29a, miR-29b1, miR-29b2, and miR-29c. Studies have found that among these subtypes, the target genes of miR-29 $\alpha$ are most associated with fibrosis (37). In our study, the expression of miR-29 $\alpha$ in BMSCs-Exo ranked among the top ten, so we chose miR-29 $\alpha$ for our research.

\section{Acknowledgments}

The present work and publication are supported by The Maternal and Child Health Research Project of Jiangsu Province (F201737, F201513).

\section{Potential Conflict of Interest}

The authors have no conflicting financial interest.

\section{References}

1. Nishi Y, Takeshita T. [Asherman syndrome]. Nihon Rinsho 2006;Suppl 2:418-421. Japanese

2. March CM. Asherman's syndrome. Semin Reprod Med 2011;29:83-94

3. Zikopoulos KA, Kolibianakis EM, Platteau P, de Munck L, Tournaye H, Devroey P, Camus M. Live delivery rates in subfertile women with Asherman's syndrome after hysteroscopic adhesiolysis using the resectoscope or the Versapoint system. Reprod Biomed Online 2004;8:720-725 
4. Thomson AJ, Abbott JA, Deans R, Kingston A, Vancaillie TG. The management of intrauterine synechiae. Curr Opin Obstet Gynecol 2009;21:335-341

5. Chen F, Duan H, Zhang Y, Wu YH. [Effect and mechanism of formation of intrauterine adhesion at different dose of estrogen]. Zhonghua Fu Chan Ke Za Zhi 2010;45:917920. Chinese

6. Deans R, Abbott J. Review of intrauterine adhesions. J Minim Invasive Gynecol 2010;17:555-569

7. Yu D, Wong YM, Cheong Y, Xia E, Li TC. Asherman syndrome--one century later. Fertil Steril 2008;89:759-779

8. Morrison SJ, Uchida N, Weissman IL. The biology of hematopoietic stem cells. Annu Rev Cell Dev Biol 1995;11:35-71

9. Bianco P, Riminucci M, Gronthos S, Robey PG. Bone marrow stromal stem cells: nature, biology, and potential applications. Stem Cells 2001;19:180-192

10. Alvarez-Buylla A, García-Verdugo JM, Tramontin AD. A unified hypothesis on the lineage of neural stem cells. Nat Rev Neurosci 2001;2:287-293

11. Gallacher L, Murdoch B, Wu D, Karanu F, Fellows F, Bhatia M. Identification of novel circulating human embryonic blood stem cells. Blood 2000;96:1740-1747

12. Du H, Taylor HS. Contribution of bone marrow-derived stem cells to endometrium and endometriosis. Stem Cells 2007;25:2082-2086

13. Nagori CB, Panchal SY, Patel H. Endometrial regeneration using autologous adult stem cells followed by conception by in vitro fertilization in a patient of severe Asherman's syndrome. J Hum Reprod Sci 2011;4:43-48

14. Johnstone RM, Adam M, Hammond JR, Orr L, Turbide C. Vesicle formation during reticulocyte maturation. Association of plasma membrane activities with released vesicles (exosomes). J Biol Chem 1987;262:9412-9420

15. Ogawa Y, Kanai-Azuma M, Akimoto Y, Kawakami H, Yanoshita R. Exosome-like vesicles with dipeptidyl peptidase IV in human saliva. Biol Pharm Bull 2008;31:10591062

16. Tkach $M$, Théry C. Communication by extracellular vesicles: where we are and where we need to go. Cell 2016;164: 1226-1232

17. Raposo G, Stoorvogel W. Extracellular vesicles: exosomes, microvesicles, and friends. J Cell Biol 2013;200:373-383

18. van Niel G, Raposo G, Candalh C, Boussac M, Hershberg R, Cerf-Bensussan N, Heyman M. Intestinal epithelial cells secrete exosome-like vesicles. Gastroenterology 2001;121: 337-349

19. Blanchard N, Lankar D, Faure F, Regnault A, Dumont C, Raposo G, Hivroz C. TCR activation of human T cells induces the production of exosomes bearing the TCR/CD3/ zeta complex. J Immunol 2002;168:3235-3241

20. Théry C, Amigorena S, Raposo G, Clayton A. Isolation and characterization of exosomes from cell culture supernatants and biological fluids. Curr Protoc Cell Biol 2006;30:3.22. 1-3.22.29

21. Conforti A, Alviggi C, Mollo A, De Placido G, Magos A. The management of Asherman syndrome: a review of literature. Reprod Biol Endocrinol 2013;11:118

22. Salazar CA, Isaacson K, Morris S. A comprehensive review of Asherman's syndrome: causes, symptoms and treatment options. Curr Opin Obstet Gynecol 2017;29:249-256

23. Berman JM. Intrauterine adhesions. Semin Reprod Med 2008;26:349-355

24. Lin HY, Wang FS, Yang YL, Huang YH. MicroRNA-29a suppresses CD36 to ameliorate high fat diet-induced steatohepatitis and liver fibrosis in mice. Cells 2019;8:1298

25. Harmanci D, Erkan EP, Kocak A, Akdogan GG. Role of the microRNA-29 family in fibrotic skin diseases. Biomed Rep 2017;6:599-604

26. Huang YH, Yang YL, Wang FS. The role of miR-29a in the regulation, function, and signaling of liver fibrosis. Int J Mol Sci 2018;19:1889

27. Timmers L, Lim SK, Arslan F, Armstrong JS, Hoefer IE, Doevendans PA, Piek JJ, El Oakley RM, Choo A, Lee CN, Pasterkamp G, de Kleijn DP. Reduction of myocardial infarct size by human mesenchymal stem cell conditioned medium. Stem Cell Res 2007;1:129-137

28. Hu J, Zhang L, Wang N, Ding R, Cui S, Zhu F, Xie Y, Sun X, Wu D, Hong Q, Li Q, Shi S, Liu X, Chen X. Mesenchymal stem cells attenuate ischemic acute kidney injury by inducing regulatory $\mathrm{T}$ cells through splenocyte interactions. Kidney Int 2013;84:521-531

29. Li T, Yan Y, Wang B, Qian H, Zhang X, Shen L, Wang M, Zhou Y, Zhu W, Li W, Xu W. Exosomes derived from human umbilical cord mesenchymal stem cells alleviate liver fibrosis. Stem Cells Dev 2013;22:845-854

30. Blazquez R, Sanchez-Margallo FM, de la Rosa O, Dalemans W, Alvarez V, Tarazona R, Casado JG. Immunomodulatory potential of human adipose mesenchymal stem cells derived exosomes on in vitro stimulated T cells. Front Immunol 2014;5:556

31. Kim DK, Nishida H, An SY, Shetty AK, Bartosh TJ, Prockop DJ. Chromatographically isolated CD63+CD81+ extracellular vesicles from mesenchymal stromal cells rescue cognitive impairments after TBI. Proc Natl Acad Sci U S A 2016;113:170-175

32. Pohlers D, Brenmoehl J, Löffler I, Müller CK, Leipner C, Schultze-Mosgau S, Stallmach A, Kinne RW, Wolf G. TGF-beta and fibrosis in different organs- molecular pathway imprints. Biochim Biophys Acta 2009;1792:746-756

33. Chen B, Li R, Yan N, Chen G, Qian W, Jiang HL, Ji C, Bi ZG. Astragaloside IV controls collagen reduction in photoaging skin by improving transforming growth factor- $\beta$ /Smad signaling suppression and inhibiting matrix metalloproteinase-1. Mol Med Rep 2015;11:3344-3348

34. Inagaki $Y$, Kushida M, Higashi K, Itoh J, Higashiyama R, Hong YY, Kawada N, Namikawa K, Kiyama H, Bou-Gharios G, Watanabe T, Okazaki I, Ikeda K. Cell type-specific intervention of transforming growth factor be$\mathrm{ta} /$ Smad signaling suppresses collagen gene expression and hepatic fibrosis in mice. Gastroenterology 2005;129:259-268

35. Xiao J, Meng XM, Huang XR, Chung AC, Feng YL, Hui DS, Yu CM, Sung JJ, Lan HY. miR-29 inhibits bleomy- 
cin-induced pulmonary fibrosis in mice. Mol Ther 2012;20: $1251-1260$

36. van Rooij E, Sutherland LB, Thatcher JE, DiMaio JM, Naseem RH, Marshall WS, Hill JA, Olson EN. Dysregulation of microRNAs after myocardial infarction reveals a role of miR-29 in cardiac fibrosis. Proc Natl Acad Sci U
S A 2008;105:13027-13032

37. Alizadeh M, Safarzadeh A, Beyranvand F, Ahmadpour F, Hajiasgharzadeh K, Baghbanzadeh A, Baradaran B. The potential role of miR-29 in health and cancer diagnosis, prognosis, and therapy. J Cell Physiol 2019;234:1928019297 